\title{
Comment le verbe voir s'est engagé sur la voie de la désémantisation
}

\author{
Raja Gmir-Ezzine \\ Faculté des Sciences Humaines et Sociales de Tunis, 94, Boulevard du 9 avril 1938, 1007, Tunis \\ raja.gmir@yahoo.fr
}

\begin{abstract}
Résumé. Dans ce travail, nous montrons que le verbe VOIR se désémantise, dans certains emplois, jusqu'à se grammaticaliser et devenir un véritable outil-grammatical de différentes diathèses en français. $\mathrm{Ce}$ processus de désémantisation est décrit à partir des emplois primaires du verbe, notamment les emplois perceptif et cognitif dans JE VOIS QUE, JE VOIS INF. De ces emplois et à partir de la construction (SE) VOIR + INF dérivent d'autres usages qui sont moins prototypiques. Cette construction permet la promotion au rang et à la fonction de sujet syntaxique, d'une part, des compléments d'objet direct et indirect et, d'autre part, des circonstants de lieu et de temps. Le locuteur peut alors, à l'aide du verbe VOIR dégradé au statut de semi-auxiliaire et dénué de tout sens perceptif, réorganiser l'ordre des mots sur la chaîne syntagmatique pour former ces diathèses.
\end{abstract}

\begin{abstract}
In this work, we show that the verb VOIR loses its meaning in some uses, until it gets grammaticalized and becomes a genuine grammatical tool of different voices in French. This process of semantic bleaching is described on the basis of the primary uses of the verb, notably the perceptual and cognitive ones in JE VOIS QUE, JE VOIS INF. These uses and the middle construction (SE) VOIR + INF are the source of alternative uses that are less prototypical. By the mean of this construction, first direct and indirect object complements and then adjuncts of place and time are promoted to the rank and function of syntactic subject. By using the verb VOIR demoted to the status of semi-auxiliary and devoid of any perceptive meaning, the speaker can rearrange the sequence of participants to produce these voices.
\end{abstract}

\section{Introduction}

Le verbe VOIR connaît une désémantisation qui fait de lui un «semi-auxiliaire » de diathèses en français, dans des emplois contemporains, notamment dans l'écrit journalistique. Il a le statut de «semi-auxiliaire», car il devient un véritable outil grammatical, « lexicalement vide » perdant ainsi des « degrés de [sa] verbalité » ${ }^{1}$.

Dans ce travail nous expliquerons comment ce verbe s'est désémantisé progressivement ${ }^{2}$. Nous montrerons, d'abord, le glissement de sens qui s'effectue de la perception visuelle à la perception cognitive, ensuite, les différences sémantiques entre $(S E)$ 
$V O I R+I n f$ d'un côté et (SE) LAISSER / (SE) FAIRE + Inf de l'autre, et la différence de statut entre eux, bien que les trois partagent certaines configurations syntaxiques. Enfin, nous montrerons que VOIR suivi d'un infinitif peut sélectionner un sujet non humain et acquiert, de ce fait, le statut de semi-auxiliaire de diathèses circonstancielles en français.

\section{VOIR : de la perception visuelle au raisonnement}

Le sens premier de VOIR est perceptif : «percevoir par le sens de la vue ». Il est transitif et de ce fait il sélectionne un COD qui peut être un groupe nominal, un pronom, un groupe infinitif $^{3}$ ou une proposition conjonctive complétive. Pour les deux derniers types de COD, il est généralement possible, mais pas toujours, de passer de l'un à l'autre avec ou sans changement de sens, avec dans la plupart des cas une modification de la configuration syntaxique de la construction infinitive.

La question est de savoir jusqu'à quel point les deux constructions qn voit que $P$ et $q n$ voit INF diffèrent sémantiquement. Pour cela, nous allons les comparer en substituant la deuxième à la première.

\subsection{Alternance sans variation sémantique majeure}

Il est possible de remplacer la construction Je vois que par la construction Je vois Inf. Les exemples ci-dessous l'attestent ${ }^{4}$.

(1) Je voyais que l'image tremblotait imperceptiblement au rythme de ma respiration, des battements de mon cœur [GUIBERT Hervé, Le protocole compassionnel, 2007, p. 260]

(2) Je vis qu'il brandissait un pistolet et repris mon ascension [LITTELL Jonathan, Les bienveillantes, 2011].

(3) Je vois que le garçon remet son livre dans son sac [GUIBERT Hervé, Le mausolée des amants, 2001] (1a) Je voyais l'image trembloter.

(2a) Je le vis brandir un pistolet.

(3a) Je vois le garçon remettre son livre dans son sac.

En effet, les constructions en je vois que $\mathrm{P}_{<\text {prés> }}$ je voyais/vis que $\mathrm{P}_{<\text {imp }>}$ exprimant une relation de simultanéité ou d'inclusion temporelle entre la perception et le déroulement d'un événement ou d'une action peuvent alterner avec la construction infinitive et ne présentent pas de variation sémantique majeure.

\subsection{Alternance douteuse}

L'alternance entre les deux constructions est acceptable pour (4a), commencer l'année exprimant une action, ce qui favorise la transformation de son sujet en complément du v. VOIR. En revanche cette transformation est douteuse pour (5a-7a) soit parce que le verbe (devenir, avoir envie de) n'exprime pas une action (5a, 7a), soit en raison du déplacement du pronom locatif $y$ (j'y vois opposer..., 6a). 
(4) Je vois que vous commencez l'année dans les meilleures dispositions [DE VIGAN Delphine, $N o$ et moi, 2007].

(5) Je vois que tu deviens enragée de notre vie [GARAT Anne-Marie, Dans la main du diable, 2006]

(6) Je vois qu'on y oppose fréquemment récit à roman, apparemment au sens de "récit non fictionnel » [GENETTE Gérard, Bardadrac, 2009]

(7) Je voyais qu'il avait encore envie de parler [LITTELL Jonathan, Les bienveillantes, 2011]. (4a) Je vous vois commencer l'année dans ...

(5a) Je te vois devenir enragée de notre vie.

(6a) J'y vois opposer fréquemment récit à roman...

(7a) Je le voyais avoir encore envie de parler.

En effet, nous perdons la perception cognitive relative au raisonnement et certains cas relèvent de la bizarrerie stylistique, comme pour l'énoncé transformé $7 \mathrm{a}$, par exemple.

\subsection{Alternance possible avec une construction adjectivale (attribut de l'objet) ou prépositionnelle}

Dans le but de retrouver le sens initial, nous remplaçons la première construction par une deuxième moyennant une structure syntaxique qui n'est pas infinitive. En $(8,11)$ l'énoncé original est déjà au passif, ce qui distingue ce cas du suivant (§1.4).

(8) Je vois que vous êtes déjà terriblement atteint, mais on va essayer de vous sortir de là [GUIBERT Hervé, Le protocole compassionnel, 2007].

(9) Je vois que, sous votre manteau, vous êtes en habit, et que, de ce pas, vous allez à quelque fête... [GARAT Anne-Marie, Dans la main du diable, 2006]

(10) Je voyais qu'il était curieux: «Ah oui? Raconte. » [LITTELL Jonathan, Les bienveillantes, 2011].

(11) Je vis qu'elles étaient vêtues de blouses blanches par-dessus des complets [LITTELL Jonathan, Les bienveillantes, 2011].

Ici, l'alternance est possible, mais elle s'effectue par le biais d'une construction adjectivale ou prépositionnelle.

\subsection{Alternance avec restructuration syntaxique stylistiquement douteuse}

Dans les exemples ci-dessous, la substitution s'effectue au moyen d'une restructuration syntaxique par une forme réduite de passif. La proposition est remplacée par un participe passé en fonction d'attribut d'objet.

(12) Je vois que vous vous intéressez au rugby. [ROUBAUD Jacques, Nous, les Moins-que-Rien, Fils aînés de Personne, 2006]

(13) Je vois que je te dégoûte, tu n'as pas besoin de te cacher. [DURAS Marguerite, Cahiers de la guerre et autres textes, 2013]

(14) Je vois que cela vous chagrine. Il y a longtemps que je ne l'ai pas entendue, moi non plus. [GARAT (8a) Je vous vois déjà terriblement atteint...

(9a) Je vous vois en habit sous votre manteau (et allant de ce pas à quelque fête)

(10a) Je le voyais curieux ...

(11a) Je les vis vêtues de blouses blanches... 
Anne-Marie, Dans la main du diable, 2006]

(15) Je vois qu'elle vous contrarie. Assez pour me (15a) Je vous vois contrarié par reprocher de l'avoir faite. [GARAT Anne-Marie, elle.

Dans la main du diable, 2006].

L'énoncé transformé (12a) est acceptable, parce que sa source est une forme réfléchie (vous vous intéressez > je vous vois intéressé). Les énoncés (13a-15a) sont stylistiquement douteux, parce que le pronom sujet dans l'extrait original se retrouve en position de complément d'agent alors que cette fonction est habituellement remplie par un $\mathrm{SN}$ et non par un pronom.

\subsection{Alternance avec substitution lexicale}

Dans les deux exemples ci-dessous, l'alternance entre les deux constructions syntaxiques ne serait possible qu'au prix d'une substitution lexicale, toujours susceptible d'altérer le sens.

(16) Je vois qu'à mon âge, je peux encore séduire, (16a) Je me vois à mon âge plaire à quelqu'un... je suis le premier étonné. encore CAPABLE de séduire. [MREJEN Valérie, Eau sauvage, 2013]

(17) Je vois que vous n'êtes pas au courant, poursuivit (17a) Je vous vois IGNORANT. Moudru. [AYMÉ Marcel, Le Vin de Paris, 1947]

\subsection{Alternance impossible}

Dans cette sous-section, la substitution entre les deux constructions est invalide pour des raisons diverses.

\subsubsection{P état}

Elle est impossible quand la proposition complétive exprime un état.

(18) Je vois que les officiers ont encore de bonnes (18a)*Je vois les officiers avoir cigarettes.

encore de bonnes cigarettes.

(19) Je vis qu'il connaissait beaucoup plus de (19a) *Je le vis connaître beaucoup mathématique encore que je ne le pensais (à la plus de mathématiques.... lecture de Bords) [ROUBAUD Jacques, Le Grand Incendie de Londres, 2016]

En effet, la substitution produit un énoncé inacceptable parce qu'elle suppose que l'acte de vision porte sur un procès non borné, ce qui est logiquement exclu.

\subsubsection{Absence de parallélisme temporel}

L'alternance est impossible dans les exemples ci-dessous :

(20) Je vis qu'elle, elle comprenait tout, savait tout, et devant ce savoir si pur j'éclatai en flammes. [LITTELL Jonathan, Les bienveillantes, 2011].

(21) Je vois que les enfants poussent aussi, ajouta-t-il en regardant le bébé. AYMÉ Marcel Aymé, Nouvelles (20a) Je la vis tout comprendre, *tout savoir ${ }^{5}$ complètes, 2002]

(21a) Je vois les enfants pousser aussi.

En effet l'énoncé transformé implique un parallélisme temporel alors que l'extrait original exprime une relation d'incidence entre un acte de vision ponctuel et une situation perçue qui excède largement ce moment (à moins qu'un circonstant de temps indique une 
vision qui se prolonge dans le temps (ex. 21b, Depuis qu'ils vivent avec moi, je vois les enfants pousser).

\subsubsection{Pas de constat sans que $\mathrm{P}$}

Dans le tableau ci-dessous, la substitution est impossible :

(22) Je vois que vous êtes charmante et sympathique, j'espère que vous allez défendre le dossier.

[MREJEN Valérie, Eau sauvage, 2013]

(23) Je vois que j'ai rendez-vous avec ma fille, avec des amis, avec ma psychanalyste, avec mon libraire

[ROBIN Régine, Cybermigrances : traversée

(22a)? Je vous vois charmante et sympathique.

(23a) ? Je me vois avoir rendezvous avec ma fille... fugitives, 2004]

En effet, les deux énoncés ne sont pas équivalents sémantiquement : alors que l'extrait original (22) exprime un constat, l'énoncé transformé (22a) donne l'impression d'un acte d'imagination, lequel est exclu de la part d'un locuteur en présence de son interlocutrice. L'extrait (23) n'est compréhensible que si le locuteur constate l'indication des rendez-vous dans son agenda et à nouveau l'énoncé transformé (23a) évoque un acte d'imagination (lequel, curieusement, serait possible à la forme négative : Je ne me vois pas avoir rendezvous avec $X=$ Je ne peux pas $m$ 'imaginer avoir ce rendez-vous)

\subsubsection{Bilan intermédiaire}

$\mathrm{Au}$ terme de cette section, nous pouvons tirer plusieurs conclusions. La première est que la construction $\mathrm{SN}: \mathrm{x}$ voit $\mathrm{SN}$ :y $\mathrm{SV}_{\text {inf }}$ n'est valide que si le $\mathrm{SV}_{\text {inf }}$ véhicule la perception d'un événement ou d'une action. La deuxième est que la construction $\mathrm{SN}: \mathrm{x}$ voit $\mathrm{SN}: \mathrm{y}$ SAdj/SPrep est en général un substitut valide de la construction $\mathrm{SN}: \mathrm{x}$ voit que ${ }_{\mathrm{P}}[\mathrm{SN}$ :y est SAdj/SPrep]. La troisième est que les principaux motifs d'invalidité de l'alternance entre $\mathrm{SN}: \mathrm{x}$ voit que $\mathrm{P}$ et $\mathrm{SN}: \mathrm{x}$ voit $\mathrm{SV}_{\text {inf }}$ sont : le statut d'état de $\mathrm{P}$, l'introduction infondée d'un parallélisme temporel et la perte du statut de constat associé à la complétive que $\mathrm{P}$.

La rection d'une construction infinitive s'avère être la porte qui a ouvert sur le processus de dégradation sémantique, soit sous l'une des deux formes :

$\square$ soit pseudo-réfléchie : N:x se voit INF/ PartPassé avec sujet humain mais pas expérient d'une perception visuelle mais témoin d'une situation qui l'affecte (cf. section 2),

soit transitive : N:x voit N:y INF avec un sujet non humain mais exerçant une fonction de cadre spatial ou temporel de l'événement ou l'action exprimée par l'infinitive (cf.section 3).

Dans les deux cas, cette dégradation sémantique n'a touché que marginalement les genres littéraires car elle concerne essentiellement le discours informatif. C'est la raison pour laquelle nous substituerons au corpus littéraire de la section 1 un corpus journalistique constitué de l'année 2008 complète de trois quotidiens nationaux (Le Figaro, Libération et Le Monde) et de deux quotidiens régionaux (Ouest-France et Sud-Ouest).

\section{Se voir INF semi-auxiliaire de diathèse passive entre se laisser INF et se faire INF}

Le verbe $S E V O I R+$ INF jouit d'un statut de semi-auxiliaire dans la mesure où il peut perdre son sens perceptif et promouvoir au rang de sujet grammatical un COD (accusatif) (24) ou un COI (datif) (25) ${ }^{6}$. Il peut être ainsi assimilé à l'auxiliaire $\hat{E} T R E$, auxiliaire du passif, pour le premier, et avoir un privilège par rapport à cet auxiliaire, celui de permettre au COI d'accéder à la fonction de sujet. 
(24) En général, l'artiste SE VOIT imposer une première partie par sa maison de disque.

(Ouest-France).

(25) [...] Le président du Conseil, Silvio Berlusconi, SE VOIT épingler par la publication d'écoutes téléphoniques qui font les délices de la presse transalpine. (Le Figaro).

Ces deux exemples peuvent être paraphrasés par la construction à diathèse active en (a), ou par la construction à diathèse passive en $(b)$, uniquement pour la promotion de l'objet et non du datif :

(24a) En général, la maison de disque impose une première partie $\dot{A}$ L'ARTISTE.

(25a) La publication d'écoutes téléphoniques épingle LE PRÉSIDENT DU CONSEIL,

Silvio Berlusconi.

(24b) *En général, l'artiste EST IMPOSÉ une première partie par la maison de disque.

(25b) Le président du Conseil, Silvio Berlusconi EST EPINGLÉ par La publication

d'écoutes téléphoniques.

Dans ces cas, il nous est possible de considérer SE VOIR comme semi-auxiliaire du passif, par comparaison à l'auxiliaire $\hat{E} T R E$, auxiliaire prototypique du passif standard français puisqu'il permet, tout comme $\hat{E} T R E$ une réorganisation des différents actants sur la chaîne syntagmatique.

Les verbes $S E$ LAISSER et $S E$ FAIRE peuvent avoir des emplois de semi-auxiliaires de différentes diathèses ${ }^{7}$ : permissive pour SE LAISSER, causative pour SE FAIRE, mais aussi passive pour les deux verbes. Ils sont à rapprocher, de ce fait, du verbe SE VOIR. En effet, tout comme lui, ils se construisent avec l'infinitif et s'apparentent à la construction du passif standard dans la répartition des participants du procès avec promotion de l'objet et dégradation de l'agent.

\subsection{SE LAISSER, SE FAIRE}

Nous allons pour le besoin de cet exposé remplacer le verbe (SE) VOIR par (SE) LAISSER et par ( $S E F A I R E$ ), pour comparer les constructions syntaxiques et voir quelle interprétation passive et quel statut ils peuvent avoir par rapport au semi-auxiliaire passif SE VOIR. En fait, nous voulons savoir s'il y a des différences entre des constructions telles que : $Q n$ me/te/le/la, etc. voit/laisse/fait INF et Qn se voit/laisse/fait INF.

(25c) [...] Le président du Conseil, Silvio Berlusconi, SE LAISSE épingler par la publication d'écoutes téléphoniques qui font les délices de la presse transalpine.

(25d) [...] Le président du Conseil, Silvio Berlusconi, SE FAIT épingler par la publication d'écoutes téléphoniques qui font les délices de la presse transalpine.

D'un point de vue syntaxique, les énoncés avec SE VOIR et ceux avec SE LAISSER/SE FAIRE ont la même construction. On a pu remplacer le premier par les seconds. D'un point de vue sémantique, ils ont la même interprétation, ils sont passifs (il s'agit, bien-sûr, d'une interprétation passive majeure, c'est-à-dire générale qui ne tient pas compte des différences sémantiques particulières de chacune des constructions). En effet ils peuvent, les uns et les autres suppléer à la construction passive canonique du français, celle avec $\hat{E} T R E+P P$ (cf. exemple 25b ci-dessus). Cependant, ils n'ont pas exactement le même sens passif puisqu'il y a une différence de nuance. Avec $S E V O I R$, l'interprétation est connective : le sujet patient est témoin, il se contente d'assister à la scène. Avec SE LAISSER, l'interprétation est permissive, c'est-à-dire que le sujet patient a une certaine volonté, il ne perd pas complètement le contrôle de la situation dans laquelle il se trouve affecté. Avec les emplois originaux de SE FAIRE + Inf, l'interprétation est instigatrice, c'est-à-dire que le sujet patient contrôle et fait que le procès ait lieu; il a le contrôle de la situation dans laquelle il se trouve affecté (ex. Marie s'est fait teindre les cheveux), et dans ses emplois 
désémantisés, les constructions est perçue comme une simple variante du passif (ex. Marie $s$ 'est fait voler son sac à main).

\subsection{1 (SE) LAISSER}

L'énoncé (forgé) $(26)^{8}$ :

(26) Sophie a laissé son frère escroquer son mari / l'a laissé escroquer son mari signifie que :

(26a) Sophie n'a pas empêché son frère / ne l'a pas empêché d'escroquer son mari.

Dans cet exemple, nous avons trois actants, avec deux sujets (à prendre au sens large) et un patient: un sujet syntaxique (Sophie) qui ne correspond pas au sujet sémantique (l'agent) (son frère) de l'action. Avec SE LAISSER nous avons deux actants où le sujet syntaxique (à la fois agent et patient) ne correspond pas à l'agent de l'action, celui-ci est introduit par la préposition par :

(27) Sophie s'est laissé escroquer par son frère.

signifie que

(27a) Sophie n'a pas empêché son frère de l'escroquer.

Une construction à deux actants comme dans (27) peut avoir une autre configuration syntaxique. En effet, nous pouvons avoir la construction à deux actants avec LAISSER ou avec SE LAISSER (28) sans changement de sens :

(28) Sophie a laissé un voleur lui arracher son sac / s'est laissé arracher son sac par un voleur.

Les deux énoncés signifient que

(28a) Sophie n'a pas empêché le voleur de lui arracher son sac.

Avec LAISSER, nous avons deux configurations syntaxiques différentes, une avec deux objets directs (28b) et une avec deux objets direct et indirect (28c) :

(28b) Sophie l'a laissé le faire. $\Leftrightarrow$ (28c) Sophie le lui a laissé faire.

Ainsi, nous avons un effet d'analogie avec FAIRE, l'exemple (28d) est impossible, mais (28e) est possible :

(28d) *Sophie l'a fait le faire. $\rightarrow(28 \mathrm{e})$ Sophie le lui a fait faire.

\subsection{2 (SE) FAIRE}

FAIRE, qui est attesté en tant que semi-auxiliaire de diathèse causative ou factitive, obéit à certaines contraintes de construction. La première est l'impossibilité d'avoir deux objets directs (26b), c'est-à-dire que le support nominal agent de l'action prédiquée par le verbe à l'infinitif doit être introduit par la préposition par (26c) :

(26b) *Sophie a fait son frère escroquer son mari.

(26c) Sophie a fait escroquer son mari par son frère.

La deuxième est, ainsi, l'impossibilité d'antéposer l'agent et de le reprendre par un clitique de l'action prédiquée par le verbe à l'infinitif (26d), et la possibilité d'antéposer le patient et de le reprendre par un clitique (26e) :

$(26 \mathrm{~d}) *($ Son frère,) Sophie l'a fait escroquer son mari $\rightarrow$ (26e) (Son mari), Sophie l'a fait escroquer par son frère.

De ce fait, avec SE FAIRE, nous avons une construction telle que (27b): $\mathrm{SN}_{(\mathrm{x})}+\mathrm{SE}$ $\mathrm{FAIRE}+\mathrm{INF}+\mathrm{SN}$ (par)

(27b) Sophie s'est fait escroquer par son frère

Et nous avons deux interprétations : une non agentive et une semi-agentive :

(a) Non agentive $=$ Sophie a été escroquée par son frère

(b) Semi-agentive = Sophie a agi de telle sorte que son frère a pu l'escroquer (elle l'a bien cherché !) 


\subsection{SE VOIR}

Avec SE VOIR, nous avons deux interprétations: d'une part, l'interprétation du sens perceptif dans la construction réfléchie, d'autre part l'absence de l'interprétation du sens perceptif, c'est-à-dire la dégradation à une simple fonction actancielle dans la construction pseudo-réfléchie.

Pour la construction réfléchie :

(29) Sur la photo, le préfet SE VOIT DÉCERNER la Légion d'honneur au valeureux pompier ${ }^{9}$.

$\rightarrow$ Il se voit en train de décerner la Légion d'honneur...

Pour la construction pseudo-réfléchie :

(30) Hier le valeureux pompier qui a perdu la vue dans un incendie $S$ 'EST VU $D E ́ C E R N E R$ la Légion d'honneur par le préfet.

$\rightarrow$ le pompier n'a pas vu le préfet lui décerner la Légion d'honneur, il a été le bénéficiaire de la décoration.

SE VOIR se démarque, aussi, de SE LAISSER et de SE FAIRE, car il peut, contrairement à eux, alterner infinitif et participe passé, dont les valeurs aspectuelles s'opposent, valeur [résultative] pour l'infinitif et [+résultative] ${ }^{10}$ pour le participe passé :

(31) L'escroc S'EST VU CONDAMNER vs. CONDAMNÉ à une lourde peine.

Cette alternance ne concerne que le sujet de VOIR issu d'un objet direct et non d'un datif (L'escroc S'EST VU OFFRIR / *OFFERT(E) une échappatoire)

Cette alternance infinitif/participe passé concerne aussi les emplois à sujet non-humain, emplois dérivés des emplois à sujet humain vus ci-dessus. En effet, le sujet non-humain peut tenir son origine de l'accusatif :

(32) Paris S'EST VU SÉLECTIONNER/CHOISIR/SÉLECTIONNÉ/CHOISI pour les JO de 2024.

ou du datif :

(33) La France S'EST VU ATTRIBUER 46000 billets.

Quand il tient son origine de l'accusatif, SE VOIR est le plus souvent suivi du participe passé $^{11}$ et rarement de l'infinitif:

(34) Rabat S'EST VU OBLIGÉ de répercuter l'envolée des cours mondiaux sur le prix du pain.

Au terme de cette section, nous pouvons dire que $S E$ VOIR n'est pas causatif bien qu'il se comporte comme SE LAISSER et SE FAIRE (ne pas agir contre) et qu'il comble peut-être un «trou »; celui de la promotion du datif au rang et à la fonction de sujet grammatical et celui de la promotion d'un sujet non-humain, promotion impossible avec $\hat{E} T R E$, LAISSER et FAIRE.

Tab. 1. La désémantisation des semi-auxiliaires.

\begin{tabular}{|l|l|l|}
\hline \multicolumn{2}{|c|}{ désémantisation } \\
\hline \multicolumn{1}{|c|}{ constante } & \multicolumn{1}{|c|}{ occasionnelle } & \multicolumn{1}{c|}{ absente } \\
\hline $\begin{array}{l}\text { SE VOIR + Inf }<\text { sens } \\
\text { passif }>\end{array}$ & SE FAIRE + Inf & SE LAISSER + Inf \\
Il s'est vu infliger une & a) Elle s'est fait teindre & Il s'est laissé arrêter sans réagir \\
amende par le juge & les cheveux [agent] & SE VOIR + Inf < $\quad$ sens \\
& Il s'est fait interpeller \\
par la police [patient] & $\begin{array}{l}\text { actif/perceptif }>\text { A la télévision, le juge s'est vu } \\
\text { prononcer la peine capitale }\end{array}$ \\
\hline
\end{tabular}




\section{Quand le référent du sujet de VOIR cesse d'être un animé}

Il arrive que VOIR sélectionne un sujet non-humain, dont le référent est un lieu (35) ou un temps (36).

(35) Mais la commune de Paimboeuf, la plus touchée par la pollution, VOIT régulièrement APPARAITTRE de nouvelles nappes d'hydrocarbures, avec leurs effluves entêtants. (Le Monde)

(36) Cette période de paix VOIT le Japon SE DÉTACHER de l'influence chinoise pour forger sa propre culture (Le Monde).

Nous parlons dans ce cas de diathèse circonstancielle ${ }^{12}$. L'énoncé peut être paraphrasé par un énoncé à diathèse active (35a) et (36a) (voir la colonne 1 des figures ci-dessous).

- Le lieu

(35a) De nouvelles nappes d'hydrocarbures apparaissent DANS LA COMMUNE DE

$P A I M B O E U F$, la plus touchée par la pollution.

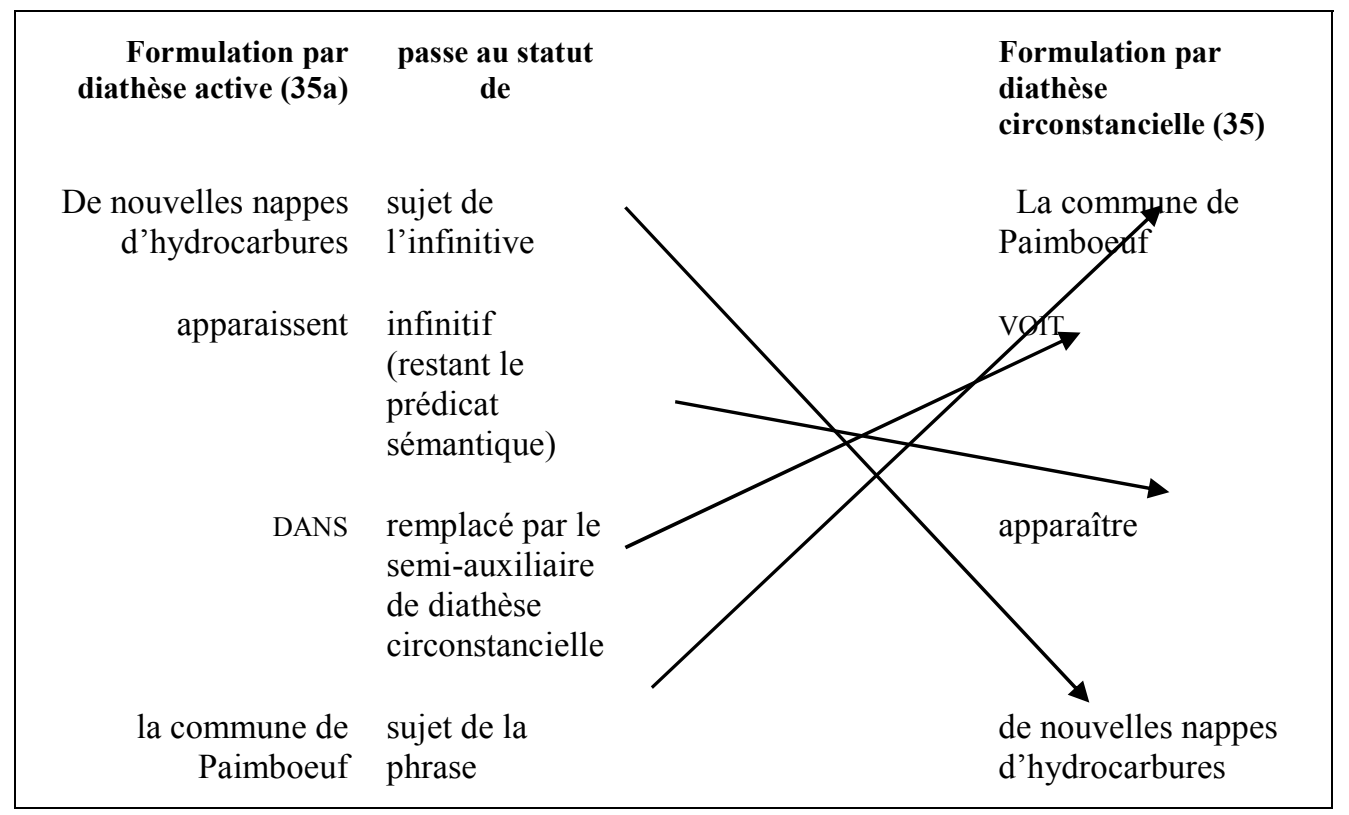

Fig. 1. De la diathèse active à la diathèse circonstancielle (LIEU).

- Le temps :

(36a) Le Japon se détache de l'influence chinoise pour forger sa propre culture PENDANT CETTE PÉRIODE DE PAIX.

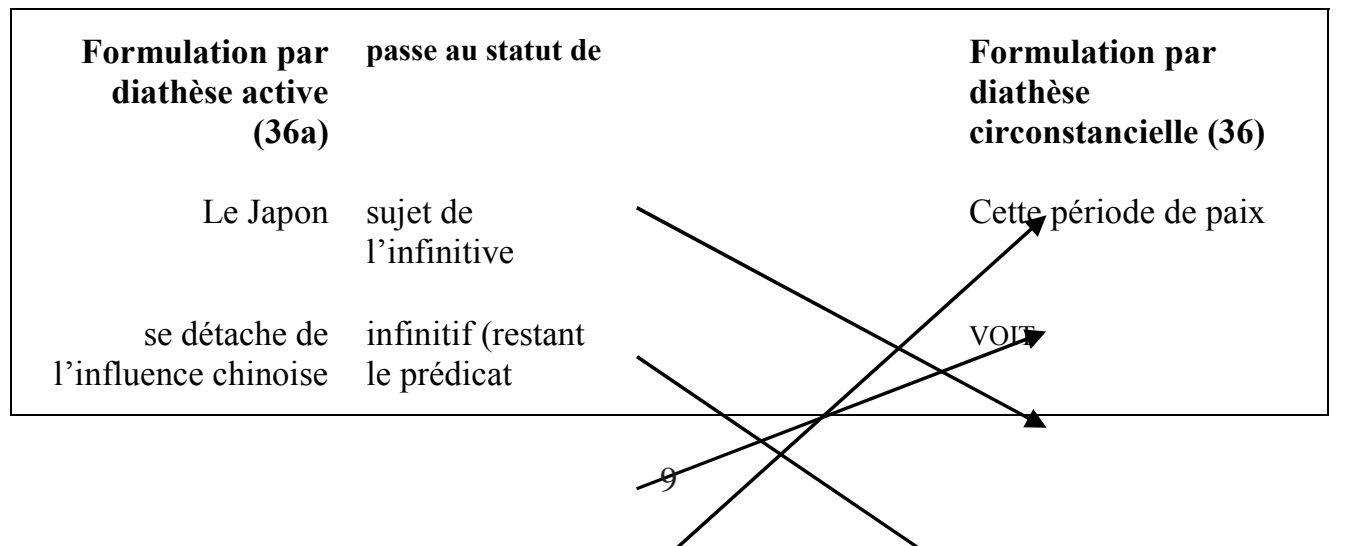




\begin{tabular}{|cll|}
\hline pendant & $\begin{array}{l}\text { sémantique) } \\
\text { remplacé par le } \\
\text { semi-auxiliaire de } \\
\text { diathèse } \\
\text { circonstancielle }\end{array}$ & le Japon \\
cette période de paix & sujet de la phrase & $\begin{array}{l}\text { se détacher de } \\
\text { l'influence chinoise }\end{array}$ \\
\hline
\end{tabular}

Fig. 2. De la diathèse active à la diathèse circonstancielle (TEMPS).

Dans les deux schémas ci-dessus, les deux phrases sont présentées verticalement, l'une à gauche, l'autre à droite. Une flèche relie les mêmes éléments se trouvant dans les deux énoncés. Cette flèche se lit de gauche à droite, c'est-à-dire que la diathèse active est l'énoncé-sa source de la diathèse circonstancielle, où ordre des mots est différent dans l'une et l'autre. En effet, la première flèche, en partant de la diathèse active, relie le sujet de la structure active De nouvelles nappes d'hydrocarbures (35a) et Le Japon (36a) au support nominal de l'infinitif dans (35) et dans (36). La deuxième relie le verbe de (35a) et (36a) à l'infinitif apparaître de (35) et se détacher de (36). La troisième flèche joint les prépositions dans (35) et pendant (36) au semi-auxiliaire VOIT qui les remplace dans l'énoncé à diathèse passive. La dernière relie enfin le SN introduit par la préposition en fonction de circonstant de lieu dans (35a) et de temps dans (36a) au sujet de la phrase à diathèse circonstancielle.

Nous pouvons dire au terme de cette sous-section que la diathèse circonstancielle est une configuration syntaxique qui permet au moyen de VOIR de redistribuer les éléments constitutifs de la diathèse de base, c'est-à-dire la diathèse active, sur la chaîne syntagmatique.

\subsection{Au-delà de l'espace et du temps}

VOIR peut sélectionner différents types de sujet, allant du sujet humain jusqu'au sujet non humain. Cette sélection traduit le processus de sa désémantisation. En effet, nous allons dans ce qui suit suivre ce processus en passant en revue les différents types de sujet que nous avons dans les deux tableaux ci-dessous : le premier trace le processus en six phases, le deuxième, dans la continuité du premier, en deux phases.

Tab. 2. Le processus de désémantisation à travers les différents types de sujet.

\begin{tabular}{|l|l|l|l|}
\hline 1. Humain & \multicolumn{2}{|l|}{ Sens perceptif } & Qn voit qn faire qch / qch se produire \\
\hline $\begin{array}{l}\text { 2. Collectivité } \\
\text { humaine }( \pm \\
\text { institutionnelle) }\end{array}$ & $\begin{array}{l}\text { Méto- } \\
\text { nymie } \\
\text { possesseur) }\end{array}$ & $\begin{array}{l}\text { (37) La fondation Hamon [qui] a vu } \\
\text { s'évaporer environ 7 millions d' } € \text { de fonds } \\
\text { publics }(L b) \rightarrow 7 \text { millions d' } € \text { de fonds } \\
\text { publics se sont évaporés de la Fondation } \\
\text { Hamon }\end{array}$ \\
\hline $\begin{array}{l}\text { 3a. Lieu } \\
\text { (-institutionnel) }\end{array}$ & $\begin{array}{l}\text { Méto- } \\
\text { nymie }\end{array}$ & $\begin{array}{l}\text { (localisé } \\
2\end{array}$ & $\begin{array}{l}\text { (38) Lalisateur) halte de St-Jean-Pied-de-Port a vu } \\
\text { passer 5500 pélerins en plus }(S O) \rightarrow 5500 \\
\text { pélerins sont passés par la halte de SJPP }\end{array}$ \\
\hline
\end{tabular}




\begin{tabular}{|l|l|l|l|}
\hline $\begin{array}{l}\text { 3b. Lieu } \\
\text { (+institutionnel) }\end{array}$ & & $\begin{array}{l}\text { (39)Le tribunal a vu défiler deux types de } \\
\text { jeunesse ...(OF) } \rightarrow \text { deux types de jeunesse } \\
\text { ont défilé devant le tribunal }\end{array}$ \\
\hline $\begin{array}{l}\text { 4. Lieu } \\
\text { englobant }\end{array}$ & $\begin{array}{l}\text { Méto- } \\
\text { nymie } \\
3\end{array}$ & $\begin{array}{l}\text { (lieu 3 } \rightarrow \\
\text { ensemble dont } \\
\text { fait partie ce } \\
\text { lieu [par } \\
\text { relation de } \\
\text { possession] }\end{array}$ & $\begin{array}{l}\text { (40)Le barrage du Bas Sabor (...) a vu SA } \\
\text { première pierre posée symboliquement }(L b) \\
\text { [+aspect résultatif] } \rightarrow \text { La première pierre } \\
\text { du barrage du BS a été posée } \\
\text { symboliquement }\end{array}$ \\
\hline 5. Temps & $\begin{array}{l}\text { Méto- } \\
\text { nymie } \\
4\end{array}$ & $\begin{array}{l}\text { (circonstance } \\
\text { temporelle } \rightarrow \\
\text { témoin } \\
\text { temporel) }\end{array}$ & $\begin{array}{l}\text { (41)La période d'avant-guerre [qui] voit } \\
\text { naitre le football professionnel en 1932 (LM } \\
\text { 2008) } \rightarrow \text { Le football professionnel naît } \\
\text { pendant la période d'avant-guerre, en 1932 }\end{array}$ \\
\hline $\begin{array}{l}\text { 6. Evénement / } \\
\text { activité }\end{array}$ & $\begin{array}{l}\text { Méto- } \\
\text { nymie } \\
5\end{array}$ & $\begin{array}{l}\text { (humain 1 } \rightarrow \\
\text { événement } \\
\text { auquel } \\
\text { participe } \\
\text { l'humain) }\end{array}$ & $\begin{array}{l}\text { (42)Ce mini-championnat [qui] voit } \\
\text { s'affronter les quatre meilleures équipes... } \\
\text { (OF) } \rightarrow \text { Les quatre meilleures équipes } \\
\text { s'affrontent dans/lors de ce mini- } \\
\text { championnat }\end{array}$ \\
\hline
\end{tabular}

Nous comptons six phases dans le processus des emplois désémantisés du verbe VOIR ; la première est celle de l'emploi propre du verbe VOIR (sens perceptif) sélectionnant un sujet humain. À partir de la phase 2, nous avons des emplois métonymiques (de 1 à 5) où le sujet sélectionné renvoie à une collectivité humaine ( \pm institutionnelle) (37), à un lieu (institutionnel) (38), (+ institutionnel) (39), à un lieu englobant (40), au temps (41) et à un événement ou une activité (42). En effet, les différents sujets non-humains soulignés dans les exemples sélectionnés sont des sujets atypiques de VOIR au sens perceptif. Ils correspondent à des circonstants de lieu ou de temps, sauf pour l'exemple (40) où l'expression du lieu englobant renvoie à une relation de possession paraphrasé par un complément du nom dans l'énoncé à diathèse active.

Pour ce qui est des deux emplois figurant dans le tableau 2 ci-dessous :

Tab. 3. Les emplois déviants.

\begin{tabular}{|l|l|l|l|}
\hline $\begin{array}{l}7 . \\
\text { Produit } \\
\text { de } \\
\text { l'esprit }\end{array}$ & $\begin{array}{l}\text { Méto- } \\
\text { nymie } \\
6\end{array}$ & $\begin{array}{l}\text { (humain } \\
\text { 1/collectivité } \\
\begin{array}{l}\text { intellectuel) } \\
\text { inoduit }\end{array}\end{array}$ & $\begin{array}{l}\text { (43) L'histoire de la Méditerranée a vu alterner des } \\
\text { périodes de relative tranquillité (...) et des périodes } \\
\text { de guerre (...) (LM) } \rightarrow \text { des périodes de ... ont alterné } \\
\text { dans l'histoire de la Méditerranée. } \\
\text { (44) Le prix Jag de Bellouet a vu la victoire de Nikel } \\
\text { de Neret avec Mlle Barthélémy (LM) } \rightarrow \text { Nikel de } \\
\text { Neret [cheval] a été victorieux avec Mlle Barthélémy } \\
\text { [écuyère] lors du prix JdB }\end{array}$ \\
\hline $\begin{array}{l}\text { Compor- } \\
\text { tement }\end{array}$ & $\begin{array}{l}\text { Méto- } \\
\text { nymie }\end{array}$ & $\begin{array}{l}\text { ( } \rightarrow \text { compor- } \\
\text { tement) }\end{array}$ & $\begin{array}{l}\text { (45) Cette pratique voit les géants de la grande } \\
\text { distribution apposer leur logo sur une liste croissante } \\
\text { de produits qu'ils n'ont pas fabriqués (LM) } \rightarrow \text { Dans } / \\
\text { avec cette pratique, les géants de la grande } \\
\text { distribution apposent leur logo.... }\end{array}$ \\
\hline
\end{tabular}


Il s'agit d'emplois métonymiques créatifs qui relèvent $\mathrm{du}$ « français exploratoire ${ }^{14}$. En effet, ces sujets sont inhabituels, voire déviants. Ils montrent le degré maximum de la désémantisation de VOIR.

$\mathrm{Au}$ terme de cette sous-section, nous pouvons dire que la désémantisation de VOIR se résume en trois grandes phases, l'une dérivant de l'autre. La première englobe les emplois 1 et 2 , la deuxième les emplois $3,4,5$ et 6 , relevant du français avancé ${ }^{15}$, la troisième les emplois 7 et 8 relevant du « français exploratoire ».

la phase 1 englobe l'emploi propre sélectionnant un sujet humain en (1) et le premier emploi désémantisé (début du processus) sélectionnant une collectivité humaine en (2). De ce dernier emploi dérive le troisième dont le sujet exprime le lieu ( \pm institutionnel). Ce troisième emploi donne naissance à deux autres, le 4 et le 5 , le lieu englobant et le temps. Du sujet temps dérive le dernier emploi de la deuxième phase, à savoir le sujet exprimant l'événement ou l'activité. La phase trois, phase du «français exploratoire », regroupe les emplois 7 et 8 dont le sujet est soit un produit de l'esprit, soit un comportement.

\section{Conclusion}

Dans ce travail, nous avons passé en revue les emplois primaires et dérivés du verbe VOIR, que nous reprenons dans la figure ci-dessous. Nous avons conclu que le verbe VOIR peut avoir, à côté de son emploi propre, des emplois grammaticalisés qui font de lui un semiauxiliaire de diathèse passive, notamment avec $S E V O I R$, mettant en position de sujet l'accusatif ou le datif, et de diathèse circonstancielle avec VOIR, mettant en position de sujet les circonstants de temps ou de lieu. Dans les deux diathèses, le semi-auxiliaire est suivi généralement de l'infinitif, tout comme les autres semi-auxiliaires LAISSER et FAIRE. Mais contrairement à eux, VOIR peut être suivi d'un participe passé notamment quand il promeut l'accusatif, ou le circonstant de temps ou de lieu. Cette construction $V O I R+P P$ nous montre que $V O I R$ acquiert le véritable statut de semi-auxiliaire, puisqu'il n'est plus verbe, n'exigeant pas l'infinitif à sa suite, si nous nous référons à la règle grammaticale disant que quand deux verbes se suivent, le deuxième se met à l'infinitif. Il ressemble, de ce fait, aux auxiliaires français prototypiques, à savoir $\hat{E} T R E$ et $A V O I R$ qui sont toujours suivis du participe passé. 


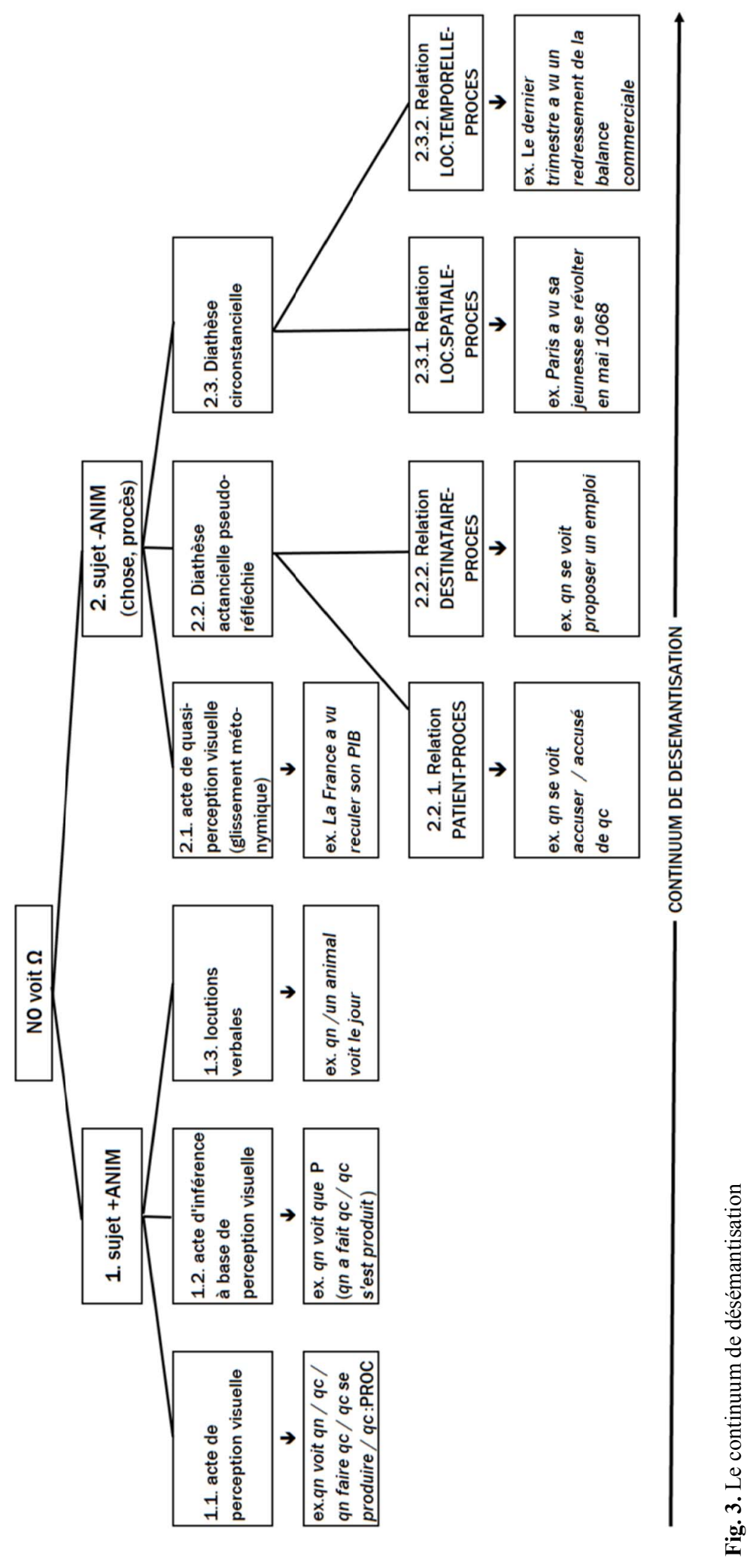




\section{Références Bibliographiques}

Arrivé, M., Gadet, F., Galmiche, M., (1986), La grammaire d'aujourd'hui. Guide alphabétique de linguistique française. Paris, Flammarion.

Blanche-Benveniste, C. (2002), Auxiliaires et degrés de « verbalité ». Syntaxe et sémantique, 2002/1 $\left(N^{\circ} 3\right)$, p. 75-97. DOI : 10.3917/ss.003.0075. URL : https://www.cairn.info/revue-syntaxe-etsemantique-2002-1-page-75.htm

Chocheyras, J., (1968), Un nouvel outil grammatical en français moderne : le verbe voir. Le Français Moderne, 3, pp.219-225.

Creissels, D. (1995), Eléments de syntaxe générale, P.U.F.

Deronne, E., (2007a), Des voix aux diathèses. Article issu d'une intervention au séminaire de l'ATILF (Nancy) de novembre 2006. Publié sur le site http://emmanuel.deronne.free.fr.

Deronne, E., (2007b), Embarquement immédiat ! Etude des diathèses autour du verbe embarquer (de l'eau) (à l'exclusion des formulations renvoyant aux relations de causalité). Publié sur le site des Archives HAL, http://halshs.archives.

Deronne, E., (2008), Embarquement différé, Seconde étude des diathèses autour du verbe embarquer (de l'eau), consacrée aux formulations renvoyant aux relations de causalité. Publié sur le site des Archives HAL, http://halshs.archives.

Dubois, J., Giacomo, M., Guespin, L., Marcellesi, C., Marcellesi, J-B., Mével, J-P., (1994), Dictionnaire de linguistique et des sciences de langage. Paris, Larousse.

François, J., (2001), Désémantisation verbale et grammaticalisation. (Se) voir employé comme outil de redistribution des actants. Syntaxe et sémantique 2, pp. 159-175.

Gaatone, D. (1970), Le rôle de voir dans les procédures de retournement de la phrase. Linguistics 58, pp.18-29.

Gaatone, D., (1998), Le passif en français. Champs linguistiques, Recherches, Editions Duculot.

Gaatone, D., (2000), Pour une définition restrictive du passif en français. Le passif, L. SchØsler (éd) pp.15-22.

GMiR-Ezzine R. 2007. L'actant circonstanciel sujet. Actes du Colloque: Morphosyntaxe et sémantique du verbe. Relations actancielles, voix, aspect et statut grammatical en français et en arabe, organisé à Tunis le 3 novembre 2006, publié dans Les Cahiers du CRISCO, 23, J. François \& A. Brahim (éds.), CRISCO, Université de Caen, CNRS, p. 50-59, téléchargeable sur le site www. crisco.unicaen.fr

GMIR-Ezzine R. 2009. Existe-t-il une voix circonstancielle en français? Les voix du français : usages et représentations, Actes du colloque d'Oxford, in l'Association for French Language Studies (dir.), Francfort/Main : Peter Lang, p. 309-318.

GMIR-Ezzine R. 2014a. La diathèse circonstancielle en français au moyen du verbe VOIR : étude syntaxique, sémantique et pragmatique. (sous la direction de M. Michel Charolles).

GMIR-EzzINE R. 2014b. Une nouvelle diathèse en français. Le français: langue une, langue plurielle, Actes du colloque de Cambridge, AFLS (dir.) Les Cahiers AFLS On-Line (URL: http://afls.net/cahiers/19.2/3-CahiersAFLS19(2)-Gmir-Ezzine.pdf)

GMiR-Ezzine R / François J. 2015. La diathèse circonstancielle et la coprédication : l'exemple de la structure N0 voit Inf N1 / N1 Inf (N2). Contribution au colloque La coprédication. Université de Nice-Sophia Antipolis [à paraître] 
GMIR-EZZINE, R (2018) [à paraître], Diathèse passive et diathèse circonstancielle en français : structures au moyen de (SE) VOIR apparentées au passif. Contribution au colloque Autour du passif en italien et dans d'autres langues, Duino-Aurisina.

Lazard, G. (1994), L'actance. Paris, P.U.F.

Lazard, G. (1997), Actance, diathèse : questions de définition. Pour engager le dialogue avec Igor Mel'čuk. Bulletin de la Société de Linguistique de Paris, Tome Tome XCII, fasc. 1, Klincksieck, pp. 115-136.

Le Bellec, C., (2014), Quand la causative et la réflexive se rencontrent... Les différentes valeurs de la construction en se faire. Congrès Mondial de Linguistique Française - CMLF 2014 SHS Web of Conferences

Le Bellec, C., (2014), La construction passive en se faire : une forme concurrente et complémentaire $\mathrm{du}$ passif canonique. In Journal of French of Language Studies, 24, pp. 203-222. DOI : $\underline{10.1017 / \mathrm{S} 0959269513000033}$

Le Bellec, C., (2015), La grammaticalisation de la construction passive en se faire. Cahiers de praxématique $65, \mathrm{mis}$ en ligne, consulté le 11 juin 2017, URL: $\mathrm{http} /$ praxematique.revues.org/4135

Muller, C., (2005), Diathèses et voix en français. Interaction entre sémantique et pragmatique, Actes du XI Séminaire de Didactique Universitaire (Constanta 2004, Université Ovidius, Association des Chercheurs en Linguistique Française), Editura ASE, Bucuresti, pp.73-95.

Muller, C., (2006), Diathèses et voix en français : une répartition des rôles. In : Grammatica, Festschrift in honour of Michael Herslund (H. Nølke, I. Baron, H. Korzen, H. K. Müller, Eds), pp. 343-359.

Riegel, M., Pellat, J-C., Rioul, R., (1994), Grammaire méthodique du français. Paris, P.U.F.

Shyldkrot, B.-Z. H., (1981), A propos de la forme passive se voir $+\mathrm{V}_{\text {inf }}$. Folia Linguistica, Acta Societatis Linguisticae Europaeae, XV/3-4, pp.387-407.

Shyldkrot, B.-Z. H., (1984a), Remarques sur la construction dite causative : faire (laisser, voir, entendre, sentir) + Infinitif, by Karl Johan Danell. Romance Philology, vol.XXXVIII, $n^{\circ} 1$, pp. 81 87.

Shyldkrot, B.-Z. H., (1984b), La concurrence entre la proposition conjonctive et voir + la proposition infinitive. French Review, Volume 58, Numéro 2, pp.202-214.

Shyldkrot, B.-Z. H. (1987), Les compléments de temps et de lieu sont-ils toujours des compléments circonstanciels ?. Folia linguistica, Acta Societatis Linguisticae Europaeae, Tome XXI/ 2-4, pp.229-247.

Shyldkrot, B.-Z. H., (1989), Les verbes de perception : étude sémantique. Actes du XVIIIe Congrès International de Linguistique et Philologie Romanes, pp.282-294.

Shyldkrot, B.-Z. H., (1997), La grammaticalisation des auxiliaires : Le cas de voir. Scolia 10, pp.205224.

Shyldkrot B.-Z. H., (1999), Analyse sémantique d'une forme passive complémentaire : se laisser. Les auxiliaires : délimitation, grammaticalisation et analyse In: Langages, $33^{\mathrm{e}}$ année, $n^{\circ} 135$, sous la direction de Hava Bat-Zeev Shyldkrot. pp. 63-74.

Tesnière, L. (1969), Eléments de syntaxe structurale. $2^{\text {ème }}$ édition revue et corrigée, Paris, Editions Klincksieck.

Veecock-Boisédu, C, (2008), Se faire + infinitif : valeurs pragmatico-énonciatives d'une construction « agentive ». Congrès Mondial de Linguistique Française - CMLF'08 ISBN 978-2-7598-0358-3, Paris, Institut de Linguistique Française Sémantique DOI 10.1051/cmlf08103, Durand J. Habert B., Laks B. (éds.), en ligne à l'adresse suivante: http://www.linguistiquefrancaise.org or http://dx.doi.org/10.1051/cmlf08103 
Wilmet, M. (2000) Du passif à la topicalisation ou pour changer de sujet. Le passif. Actes du colloque, in. Schøsler, L. (éd.)

${ }^{1}$ Claire Blanche-Benveniste 2002, p. 75.

${ }^{2}$ Ni le TLF (article voir, rubrique Histoire et étymologie), ni le Dictionnaire Historique de la Langue Française (1992), n'illustrent l'emploi du v. voir comme semi-auxiliaire d'une forme de passif. Le dictionnaire de Littré (article voir, rubrique 39) mentionne "Se voir, suivi d'un verbe actif à l'infinitif, être ce qu'indique ce verbe." et l'illustre par Ce qui désespérait le plus de si braves hommes, c'était de se voir assommer comme des bêtes prises dans un piège, sans pouvoir venger leur mort, [Vaugelas, Quinte-Curse]. Quant à l'emploi à sujet locatif (spatial ou temporel) il n'est mentionné dans aucun des trois dictionnaires, ce qui suggère que cet emploi est extrêmement récent.

3 Nous ne tenons compte ici que de l'analyse grammaticale traditionnelle. Celle-ci considère qu'à la suite de VOIR nous avons une proposition infinitive composée d'un verbe à l'infinitif ayant son propre sujet, et que le tout fonctionne comme COD du verbe perceptif. Cette analyse pose effectivement des problèmes sur lesquels nous n'allons pas nous attarder ici et que nous avons soulevés dans un travail antérieur (cf. Raja Gmir-Ezzine 2014).

${ }^{4}$ Les exemples de cette première partie sont extraits de la base textuelle FRANTEXT (1940-2016).

${ }^{5}$ Comprendre est compatible avec une interprétation inchoative alors que savoir ne l'est pas, d'où l'astérisque.

6 Jacques François 2001.

7 Martin Riegel et alii 1994, Hava Bat-Zeev Shyldkrot 1984a, 1997,1999, Christel Le Bellec 2009, 2014, 2015, Sophie Raineri 2010, Candace Veecock-Boisédu (2008).

${ }^{8}$ Les exemples 26-28 sont des énoncés fabriqués, car nous nous basons sur le corpus journalistique uniquement pour les énoncés avec $(S E)$ VOIR qui fait l'objet de notre travail.

${ }^{9}$ Là aussi les exemples sont fabriqués, car nous n'avons pas rencontré des énoncés dans le corpus journalistique qui répondent aux besoins de l'analyse de cette sous-section.

${ }^{10}$ À prendre dans le sens d'inaccompli pour la première valeur et d'accompli pour la deuxième.

${ }^{11}$ Raja Gmir-Ezzine (2018) à paraître.

${ }^{12}$ Raja Gmir-Ezzine 2014a.

${ }^{13}$ Nous pouvons avoir après VOIR non un infinitif, mais un nom prédicatif.

${ }^{14}$ Jacques François parle de « français exploratoire ». Par « français exploratoire », l'auteur entend des structures qui peuvent disparaître et ne plus se développer.

15 Dominique Legallois parle de «français avancé ». Par «français avancé », l'auteur entend des structures non canoniques qui se développent et s'emploient de plus en plus. 